JIRSS (2020)

Vol. 19, No. 02, pp 175-204

DOI:10.52547/jirss.19.2.175

\title{
An Updated Review of Goodness of Fit Tests Based on Entropy
}

\author{
Hadi Alizadeh Noughabi ${ }^{1}$, Gholam Reza Mohtashami Borzadaran ${ }^{2}$. \\ ${ }^{1}$ Department of Statistics, University of Birjand, Birjand, Iran. \\ ${ }^{2}$ Department of Statistics, Ferdowsi University of Mashhad, Mashhad, Iran.
}

Received: 27/10/2018, Revision received: 09/09/2020, Published online: 03/02/2021

\begin{abstract}
Different approaches to goodness of fit (GOF) testing are proposed. This survey intends to present the developments on Goodness of Fit based on entropy during the last 50 years, from the very first origins until the most recent advances for different data and models. Goodness of fit tests based on Shannon entropy was started by Vasicek in 1976 and were continued by many authors. In this paper, we describe different GOF tests constructed by authors from the beginning to now. First, the problem of GOF and different types of GOF are stated. Then, the method of GOF tests based on entropy for complete and censored data is explained and all works proposed by authors in this subject are mentioned.
\end{abstract}

Keywords. Goodness of Fit Tests, Entropy, Monte Carlo Comparisons.

MSC: 62G10, 62G20.

\section{Introduction}

The term goodness of fit (GOF) was coined by Pearson in 1902, and refers to statistical tests that check the quality of a model or a distribution's fit to a set of data. The first measure of GOF for general distributions was derived by Kolmogorov (1933). Andrei Nikolaevich Kolmogorov (1905-1987), perhaps the most accomplished and celebrated Soviet mathematician of all time, made fundamental contributions to probability theory, including test statistics for distribution functions - some of which bear his name.

Corresponding Author: Hadi Alizadeh Noughabi (alizadehhadi@birjand.ac.ir)

Gholam Reza Mohtashami Borzadaran (grmohtashami@um.ac.ir)

*This work is dedicated to Professor Nasser Reza Arghami, who was one of the pioneers in goodness of fit tests based on some measures in information theory. He died in 26 July 2017 at the age of 68. 
Nikolai Vasil'yevich Smirnov (1900-1966), another Soviet mathematician, extended Kolmogorov's results to two-sample cases.

The Kolmogorov statistic (sometimes called the Kolmogorov-Smirnov test statistic test statistic, which is defined as)

$$
K S=\sup _{t}\left|F_{n}(t)-F(t)\right|,
$$

provides nonparametric GOF tests for many distributions. Here, $F$ is the cumulative distribution function of the reference distribution and $F_{n}$ is the empirical distribution function of the sample. This statisitc measures how good a parametric model fits an observed sample. The fitting problem can be split into three main steps:

- choose a suitable theoretical model,

- estimate the model parameters,

- determine the significance level.

In assessing whether a given distribution is suited to a data-set, the following tests and their underlying measures of fit can be used:

Chi-Square GOF test determines how well theoretical distribution (such as normal, binomial, or Poisson) fits the empirical distribution.

In Chi-Square GOF test, the range of the theoretical distribution is divided into a finite number of intervals. Then the numbers of points that fall into the intervals are compared, with the expected numbers of points in each interval. The Chi-Square test statistic is

$$
\chi^{2}=\frac{(O-E)^{2}}{E}
$$

where, $\chi^{2}$ is the Chi-Square GOF test, $\mathrm{O}$ stands for the observed value and E denotes the expected value. About the details of the number of intervals, on can see Greenwood and Nikulin (1996).

An important problem in statistics is to obtain information about the form of the population from which the sample is drawn. GOF tests are employed to determine how well the observed sample data "fits" some proposed model. GOF tests can be constructed based on different methods. Here, we state these methods and researchers which have been worked in this field.

\section{GOF tests based on the empirical distribution function (EDF)}

- Cramer-von Mises (1931);

- Kolmogorov-Smirnov (1933);

- Anderson-Darling (1952); 
- Kuiper (1960);

- Watson (1961);

- Lilliefors (1967);

- Zhang (2002).

2. GOF tests based on measures of the moments

- D'Agostino and Pearson (1973);

- Jarque-Bera (1980);

- Doornik-Hansen (1994);

- Bonett and Seier (2002);

- Gel and Gastwirth (2008).

3. GOF tests based on regression and correlation

- Shapiro-Wilk (1965);

- Shapiro and Francia (1972);

- Filliben (1975);

- Chen and Shapiro (1995);

- Rahman and Govindarajulu (1997).

4. GOF tests based on the empirical characteristic function

- Epps and Pulley (1983).

5. GOF tests based on the empirical likelihood ratio (ELR)

- Vexler and Gurevich (2010);

- Gurevich and Vexler (2011);

- Vexler et al. (2014).

6. GOF tests based on information measures

- These tests are completely discussed in the next section.

In numerous applications in reliability studies and engineering and management sciences, it is very important to test whether the underlying distribution has a particular form. Most statistical methods assume an underlying distribution in the derivation of their results. However, when we assume that our data follow a specific distribution, we take a serious risk. If our assumption is wrong, then the obtained results may be invalid. For example, the confidence levels of the confidence intervals or error probabilities of 
tests of hypotheses implemented may be completely off. The consequences of misspecifying the distribution may prove very costly. To avoid this problem, a formal assessment of the distributional assumption is needed.

Obviously, the specialized tests will be more powerful than an omnibus test such as the Kolmogorov-Smirnov test. D'Agostino and Stephens (1986) warned that for testing for normality, the Kolmogorov-Smirnov test is only a historical curiosity. It should never be used. It has poor power in comparison to [specialized tests such as Shapiro-Wilk, D'Agostino-Pearson, Bowman-Shenton. and Anderson-Darling tests].

\section{GOF Tests Based on Entropy}

Entropy is a measure of the unpredictability of the state, or equivalently, of its average information content. To get an intuitive understanding of these terms, consider the example of a political poll. Usually, such polls happen because the outcome of the poll is not already known. In other words, the outcome of the poll is relatively unpredictable, and actually performing the poll and learning the results gives some new information; these are just different ways of saying that the a priori entropy of the poll results is large. Now, consider the case that the same poll is performed a second time shortly after the first poll. Since the result of the first poll is already known, the outcome of the second poll can be predicted well and the results should not contain much new information; in this case the priori entropy of the second poll result is small relative to that of the first.

The development of the idea of entropy of random variables and processes by Claude Shannon provided the beginnings of information theory and of the modern age of ergodic theory. It is seen that entropy and related information measures provide useful descriptions of the long term behavior of random processes and that this behavior is a key factor in developing the coding theorems of information theory. In this article, we investigate the case of GOF based on entropy.

Suppose that a random variable $X$ has a distribution function $F(x)$ with a continuous density function $f(x)$. The entropy $H(f)$ of the random variable was defined by Shannon (1948) to be

$$
H(f)=-\int_{-\infty}^{\infty} f(x) \log f(x) d x
$$

The problem of estimation of $H(f)$ has been considered by many authors. For discrete random variables, this problem is studied by Dobrushin (1958), Hutcheston and Shenton (1974), Vatutin and Michailov (1995) and, for absolutely continuous random variables, by Vasicek (1976), Dudewicz and van der Meulen (1987), Joe (1989), Hall and Morton (1993), Van Es (1992), Correa (1995), Wieczorkowski and Grzegorewski (1999), Bowman, (1992), Ebrahimi et al. (1994), Beirlant et al. (1997), Song (2000), Goria et al. (2005), Yousefzadeh and Arghami (2008), Zamanzade and Arghami (2009, 2011), 
Alizadeh Noughabi (2010), Alizadeh Noughabi and Arghami (2010), and Alizadeh Noughabi (2015a, b).

Among these various entropy estimators, Vasicek's sample entropy has been most widely used in developing entropy-based statistical procedures (Dudewicz and van der Meulen (1981); Gokhale (1983); Arizona and Ohta (1989); Ebrahimi et al. (1992), etc).

Vasicek (1976), for one dimensional distributions, proposed an estimator of entropy. His estimate was based on the fact that $H(f)$ can be expressed as

$$
H(f)=\int_{0}^{1} \log \left\{\frac{d}{d p} F^{-1}(p)\right\} d p .
$$

The estimate was constructed by replacing the distribution function $F$ by the empirical distribution function $F_{n}$, and using a difference operator instead of the differential operator. The derivative of $F^{-1}(p)$ is then estimated by a function of the order statistics.

Assuming that $X_{1}, \ldots, X_{n}$ is the sample, the Vasicek estimator (1976) is given by

$$
H V_{m n}=\frac{1}{n} \sum_{i=1}^{n} \log \left\{\frac{n}{2 m}\left(X_{(i+m: n)}-X_{(i-m: n)}\right)\right\},
$$

where $m$ is a positive integer, $m \leq \frac{n}{2}$, and $X_{(1: n)} \leq X_{(2: n)} \leq \ldots \leq X_{(n: n)}$ are order statistics and $X_{(i: n)}=X_{(1: n)}$ if $i<1, X_{(i: n)}=X_{(n: n)}$ if $i>n$.

Vasicek proved that $H V_{m n} \rightarrow H(f)$ in probability as $n \rightarrow \infty, m \rightarrow \infty, \frac{m}{n} \rightarrow 0$.

\subsection{Test for Normal Distribution}

A well-known theorem of information theory (Shannon (1949), p. 55) states that among all distributions that possess a density function $f$ and have a given variance $\sigma^{2}$, the entropy $H(f)$ is maximized by the normal distribution. Based on this property, Vasicek (1976) introduced the following statistic for testing normality:

$$
T V_{m n}=\frac{\exp \left\{H V_{m n}\right\}}{\hat{\sigma}}=\frac{n}{2 m \hat{\sigma}}\left[\prod_{i=1}^{n}\left(X_{(i+m: n)}-X_{(i-m: n)}\right)\right]^{1 / n},
$$

where

$$
\hat{\sigma}=S=\sqrt{\frac{1}{n} \sum_{i=1}^{n}\left(X_{i}-\bar{X}\right)^{2}} .
$$

Arizona and Ohta (1989) showed that the above test statistic can be derived based on Kullback-Leibler(KL) information. Let

$$
g(x ; \mu, \sigma)=\frac{1}{\sqrt{2 \pi} \sigma} \exp \left\{-\frac{1}{2}\left(\frac{x-\mu}{\sigma}\right)^{2}\right\}
$$


KL information $f$ from $g$ can be written as

$$
\begin{aligned}
D(f, g) & =\int_{-\infty}^{\infty} f(x) \log (f(x)) d x-\int_{-\infty}^{\infty} f(x) \log (g(x ; \theta)) d x \\
& =-H(f)-\int_{-\infty}^{\infty} f(x) \log (g(x ; \theta)) d x
\end{aligned}
$$

where $H(f)$ is entropy of $f$. By replacing $g(x)$, we have

$$
D(f, g)=-H(f)+\log \sqrt{2 \pi \sigma^{2}}+\frac{1}{2} \int_{-\infty}^{\infty}\left(\frac{x-\mu}{\sigma}\right)^{2} f(x) d x .
$$

Consequently, an estimate of $D(f, g)$ is constructed as

$$
I_{m n}=\log \left[\sqrt{2 \pi \hat{\sigma}^{2}} \exp \left\{\frac{1}{2 n} \sum_{i=1}^{n}\left(\frac{x_{i}-\hat{\mu}}{\hat{\sigma}}\right)^{2}\right\} / \exp \left\{H V_{m n}\right\}\right],
$$

where $\hat{\mu}$ and $\hat{\sigma}$ are the maximum likelihood estimators of $\mu$ and $\sigma$, respectively. Thus, the test statistic due to Arizona and Ohta (1989) is given by

$$
K L_{m n}=\sqrt{2 \pi} / \exp \left\{I_{m n}\right\}
$$

It is easy to show that

$$
K L_{m n}=T V_{m n} / \sqrt{e} \text {, }
$$

where $T V_{m n}$ is the test statistic introduced by Vasicek (1976). Therefore, the KL based test for the normality is equivalent to the entropy-based test given by Vasicek (1976). Moreover, some authors presented other versions of the Vasicek's test. Park (1999) proposed a GOF for normality, based on the sample entropy of order statistics. His test statistic is

$$
D V(J, n, m)=\frac{1}{n} \sum_{i=1}^{n} \log \left(\frac{n}{2 m}\left(X_{(i+m: n)}-X_{(i-m: n)}\right)\right) J\left(u_{i}\right),
$$

and he took $k=2$ and $J(u)=2(1-2 u)$.

Choi (2008) used the sample variance $\frac{1}{n} \sum_{i=1}^{n}\left(X_{i}-\bar{X}\right)^{2}$ and concluded that the power of the Vasicek test increases and therefore improved the Vasicek's test. Also, Gurevich and Davidson (2008) indroduced the standarized forms of tests based on KL information.

The authors including Zamanzade and Arghami (2009, 2012), Alizadeh Noughabi (2010), Alizadeh Noughabi and Arghami (2011c), and Lee (2013), proposed normality tests based on new estimators of entropy. Their test statistics can be found in their papers.

Using entropy of transformed data Alizadeh Noughabi and Arghami (2013) introduced a test for normal distribution. Summary of the test is as

$$
X_{1}, \ldots, X_{n} \rightarrow Y_{i j}=X_{(i: n)}+X_{(j: n)}, i<j \rightarrow T A_{m n}=\frac{n^{\prime}}{2 m \hat{\sigma}}\left[\prod_{i=1}^{n^{\prime}}\left(Y_{(i+m)}-Y_{(i-m)}\right)\right]^{1 / n^{\prime}}
$$


where $n^{\prime}=n(n-1) / 2$ and $\hat{\sigma}=\sqrt{\frac{1}{n^{\prime}} \sum_{i=1}^{n^{\prime}}\left(Y_{i}-\bar{Y}\right)^{2}}$

\subsection{Test for Exponential Distribution}

Gokhale (1983) introduced a test for exponentaility based on maximum entropy characterization. Ebrahimi et al. (1992) proposed the following test statistic, based on KL information, for testing exponentiality:

$$
T E_{m n}=-H V_{m n}+\log \bar{X}+1,
$$

where

$$
\bar{X}=\frac{1}{n} \sum_{i=1}^{n} X_{i}
$$

Choi et al. (2004) introduced two versions of the above test using two different entropy estimators:

$$
T V E_{m n}=\exp \left(H V E_{m n}\right) / \exp (\log (\bar{X})+1)
$$

where

$$
H V E_{m n}=\frac{1}{n-m} \sum_{i=1}^{n-m}\left(\frac{n+1}{m}\left(X_{(i+m: n)}-X_{(i: n)}\right)\right)+\sum_{k=m}^{n} \frac{1}{k}+\log (m)-\log (n+1),
$$

is Van Es entropy estimator (1992) and

$$
T C_{m n}=\exp \left(H C_{m n}\right) / \exp (\log (\bar{X})+1),
$$

where $H C_{m n}$ is Correa entropy estimator (1995) given by

$$
H C_{m n}=-\frac{1}{n} \sum_{i=1}^{n} \log \left(\frac{\sum_{j=i-m}^{i+m}\left(X_{(j: n)}-\bar{X}_{(i: n)}\right)(j-i)}{n \sum_{j=i-m}^{i+m}\left(X_{(j: n)}-\bar{X}_{(i: n)}\right)^{2}}\right) .
$$

Then, Alizadeh Noughabi and Arghami (2011b) introduced two version of entropy test for exponentiality. Their tests are as

$$
T E_{m n}=\exp \left(H E_{m n}\right) / \exp (\log (\bar{X})+1),
$$

where $H E_{m n}$ is Ebrahimi et al. (1994)'s sample entropy defined as

$$
H E_{m n}=\frac{1}{n} \sum_{i=1}^{n} \log \left\{\frac{n}{c_{i} m}\left(X_{(i+m: n)}-X_{(i-m: n)}\right)\right\},
$$


where

$$
\begin{gathered}
c_{i}=\left\{\begin{array}{cc}
1+\frac{i-1}{m} & 1 \leq i \leq m, \\
2 & m+1 \leq i \leq n-m, \\
1+\frac{n-i}{m} & n-m+1 \leq i \leq n,
\end{array}\right. \\
T A_{m n}=\exp \left(H A_{m n}\right) / \exp (\log (\bar{X})+1),
\end{gathered}
$$

where $T A_{m n}$ is Alizadeh Noughabi (2010)'s estimator of entropy given by

$$
H A_{m n}=-\frac{1}{n} \sum_{i=1}^{n} \log \left\{\frac{\hat{f}\left(X_{(i+m: n)}\right)+\hat{f}\left(X_{(i-m: n)}\right)}{2}\right\},
$$

and

$$
\hat{f}\left(X_{i}\right)=\frac{1}{n h} \sum_{j=1}^{n} k\left(\frac{X_{i}-X_{j}}{h}\right),
$$

and the kernel function is set to be the standard normal density function and the bandwidth $h$ is chosen to be the normal optimal smoothing formula, $h=1.06 \mathrm{sn}^{-\frac{1}{5}}$, where $s$ is the sample standard deviation.

Park and Park (2003) constructed tests for normal and exponential distribution based on the correcting moments of the entropy estimators. They first derived the underlying nonparametric density function of $H V_{m n}$ as

$$
g_{v}(x)= \begin{cases}0 & x<\xi_{1} \text { or } x>\xi_{n+1}, \\ \frac{1}{n} \frac{2 m}{x_{(i+m: n)}-x_{(i-m: n)}} & \xi_{i}<x \leq \xi_{i+1}, \quad i=1, \ldots, n,\end{cases}
$$

where $\xi_{i}=\left(x_{(i-m: n)}+\cdots+x_{(i+m-1: n)}\right) / 2 m$.

The nonparametric distribution function of $H E_{m n}$ has been derived by Park and Park (2003) as

$$
g_{e}(x)=\left\{\begin{array}{lc}
0 & x<\eta_{1} \text { or } x>\eta_{n+1} \\
\frac{1}{n} \frac{1}{\eta_{i+1}-\eta_{i}} & \eta_{i}<x \leq \eta_{i+1}, \quad i=1, \ldots, n
\end{array}\right.
$$

where

$$
\eta_{i}=\left\{\begin{array}{lr}
\xi_{m+1}-\sum_{k=i}^{m} \frac{1}{m+k-1}\left(x_{(m+k: n)}-x_{(1: n)}\right) & \text { if } 1 \leq i \leq m, \\
\frac{\left(x_{(i-m: n)}+\cdots+x_{(i+m-1: n)}\right)}{2 m} & \text { if } m+1 \leq i \leq n-m+1, \\
\xi_{n-m+1}+\sum_{k=n-m+2}^{i} \frac{1}{n+m-k+1}\left(x_{(n: n)}-x_{(k-m-1: n)}\right) & \text { if } n-m+2 \leq i \leq n+1 .
\end{array}\right.
$$

Then, Park and Park (2003) introduced the following test statistics for testing normality.

$$
T V_{m n}=-H V_{n m}+\log \left(\sqrt{2 \pi} \hat{\sigma}_{v}\right)+\frac{1}{2}
$$




$$
T E_{m n}=-H E_{n m}+\log \left(\sqrt{2 \pi} \hat{\sigma}_{e}\right)+\frac{1}{2}
$$

where $\hat{\sigma}_{v}=\operatorname{Var}_{g_{v}}(X)$ and $\hat{\sigma}_{e}=\operatorname{Var}_{g_{e}}(X)$.

Based on other estimators, Yousefzadeh and Arghami (2008), Zamanzade and Arghami (2009), and Alizadeh Noughabi and Arghami (2011a) introduced some other tests as follows:

$$
\begin{gathered}
T Y_{m n}=-H Y 2_{m n}+\log \left(\sqrt{2 \pi} \hat{\sigma}_{y}\right)+\frac{1}{2^{\prime}} \\
T Z_{m n}=-H Z_{m n}+\log \left(\sqrt{2 \pi} \hat{\sigma}_{Z}\right)+\frac{1}{2^{\prime}} \\
T A_{m n}=-H A_{m n}+\log \left(\sqrt{2 \pi} \hat{\sigma}_{a}\right)+\frac{1}{2},
\end{gathered}
$$

where $\hat{\sigma}_{y}=\operatorname{Var}_{g_{y}}(X), \hat{\sigma}_{z}=\operatorname{Var}_{g_{z}}(X)$ and $\hat{\sigma}_{a}=\operatorname{Var}_{g_{a}}(X)$. Also,

$$
g_{y}(x)=\left\{\begin{array}{l}
0 \quad \zeta_{n+1}<x \text { or } x<\zeta_{1}, \\
\frac{\hat{F}_{y}\left(x_{(i+m: n)}\right)-\hat{F}_{y}\left(x_{(i-m: n)}\right)}{x_{(i+m: n)}-x_{(i-m: n)}} \zeta_{i}<x \leq \zeta_{i+1}, i=1, \ldots . n,
\end{array}\right.
$$

where

$$
\begin{gathered}
\zeta_{i}=\frac{x_{(i-m: n)}+\cdots+x_{(i+m-1: n)}}{\sum_{i=1}^{n}\left(\hat{F}_{y}\left(x_{(i+m: n)}\right)-\hat{F}_{y}\left(x_{(i-m: n)}\right)\right)}, i=1, \ldots . n, \\
g_{Z}(x)= \begin{cases}0 \zeta_{n+1}<x \text { or } x<\zeta_{1}, \\
\frac{1}{n\left(\eta_{i+1}-\eta_{i}\right)} \quad \zeta_{i}<x \leq \zeta_{i+1}, i=1, \ldots . n,\end{cases}
\end{gathered}
$$

and

$$
\begin{gathered}
\eta_{i}=\left\{\begin{array}{cc}
\zeta_{m+1}-\sum_{k=i}^{m} \frac{1}{k}\left(x_{(m+k: n)}-x_{(1: n)}\right) & \text { if } 1 \leq i \leq m, \\
\frac{\left(x_{(i-m: n)}+\cdots+x_{(i+m-1: n)}\right)}{2 m} & \text { if } m+1 \leq i \leq n-m, \\
\zeta_{n-m+1}+\sum_{k=n-m+2}^{i} \frac{1}{n-k+2}\left(x_{(n: n)}-x_{(k-m-1: n)}\right) & \text { if } n-m+1 \leq i \leq n+1,
\end{array}\right. \\
g_{a}(x)=\left\{\begin{array}{lc}
0 & x<\eta_{1} \text { or } x>\eta_{n+1}, \\
\frac{1}{n} \frac{1}{\eta_{i+1}-\eta_{i}} & \eta_{i}<x \leq \eta_{i+1}, \quad i=1, \ldots, n,
\end{array}\right.
\end{gathered}
$$

and

$$
\eta_{i}=\left\{\begin{array}{lr}
\zeta_{m+1}-\frac{1}{m} \sum_{k=i}^{m}\left(x_{(m+k: n)}-x_{(1: n)}\right) & \text { if } 1 \leq i \leq m, \\
\frac{\left(x_{(i-m: n)}+\cdots+x_{(i+m-1: n)}\right)}{2 m} & \text { if } m+1 \leq i \leq n-m, \\
\zeta_{n-m+1}+\frac{1}{m} \sum_{k=n-m+2}^{i}\left(x_{(n: n)}-x_{(k-m-1: n)}\right) & \text { if } n-m+1 \leq i \leq n+1 .
\end{array}\right.
$$


Similarly, Park and Park (2003) proposed two tests for exponentiality based on the correcting moments of entropy estimators. Their test statistics are

$$
\begin{aligned}
& T P 1_{n m}=-H V_{n m}+\log \left(\hat{\theta}_{v}\right)+1, \\
& T P 2_{n m}=-H E_{n m}+\log \left(\hat{\theta}_{e}\right)+1,
\end{aligned}
$$

where $\hat{\theta}_{v}=E_{g_{v}}(X)$ and $\hat{\theta}_{e}=E_{g_{e}}(X)$.

Next, Yousefzadeh and Arghami (2008), Zamanzade and Arghami (2009), and Alizadeh Noughabi and Arghami (2011a) used this idea and introduced other tests for exponentaility based on the correcting moments of entropy estimators. Their test statistics are

$$
\begin{aligned}
& T Y_{m n}=-H Y 2_{m n}+\log \left(\hat{\theta}_{y}\right)+1, \\
& T Z_{m n}=-H Z_{m n}+\log \left(\hat{\theta}_{Z}\right)+1, \\
& T A_{m n}=-H A_{m n}+\log \left(\hat{\theta}_{a}\right)+1,
\end{aligned}
$$

where $\hat{\theta}_{y}=E_{g_{y}}(X), \hat{\theta}_{z}=E_{g_{z}}(X)$ and $\hat{\theta}_{a}=E_{g_{a}}(X)$.

Based on some characterizations of the exponential distribution and entropy of transformed data, Alizadeh Noughabi and Arghami (2011d, e) constructed some tests for exponentiality. They first proved the following theorem.

Theorem 2.1. Let $X_{1}$ and $X_{2}$ be two independent observations from a continuous distribution F. Then

i) $W=\frac{X_{1}}{X_{1}+X_{2}}$ is distributed as $U(0,1)$ if and only if $F$ is exponential.

ii) $Y=\frac{X_{1}}{X_{2}}$ is distributed as $F_{(2,2)}$ if and only if $F$ is exponential.

iii) $Z=\frac{X_{1}-X_{2}}{X_{1}+X_{2}}$ is distributed as $U(-1,1)$ if and only if $F$ is exponential.

$\left(F_{(2,2)}\right.$ is Fisher distribution with 2 and 2 degrees of freedom $)$. follows.

Then, they proposed some tests for exponentaility. Summary of these tests are as

$$
\begin{aligned}
& X_{1}, \ldots, X_{n} \rightarrow W_{i j}=\frac{X_{i}}{X_{i}+X_{j}}, i \neq j \rightarrow T_{1}=-\frac{1}{n^{\prime}} \sum_{i=1}^{n^{\prime}} \log \left\{\frac{n^{\prime}}{2 m}\left(W_{(i+m)}-W_{(i-m)}\right)\right\}, \\
& X_{1}, \ldots, X_{n} \rightarrow Y_{i j}=\frac{X_{i}}{X_{j}}, i \neq j \rightarrow T_{2}=-\frac{1}{n^{\prime}} \sum_{i=1}^{n^{\prime}} \log \left\{\frac{n^{\prime}}{2 m}\left(Y_{(i+m)}-Y_{(i-m)}\right)\right\} \\
& +\frac{2}{n^{\prime}} \sum_{i=1}^{n^{\prime}} \log \left(1+Y_{i}\right), \\
& X_{1}, \ldots, X_{n} \rightarrow Z_{i j}=\frac{X_{i}-X_{j}}{X_{i}+X_{j}}, i \neq j \rightarrow T_{3}=-\frac{1}{n^{\prime}} \sum_{i=1}^{n^{\prime}} \log \left\{\frac{n^{\prime}}{2 m}\left(Z_{(i+m)}-Z_{(i-m)}\right)\right\}+\log (2),
\end{aligned}
$$

where $n^{\prime}=n(n-1)$. 


\subsection{Test for Uniform Distribution}

For the class of continuous distributions $f$, concentrated on $[0,1]$, it holds

$$
H(f) \leq 0,
$$

with the maximum value of $H(f)$, zero, being uniquely attained by the $U(0,1)$ density.

Dudewicz and van der Meulen (1981) used the above property and constructed a test of uniformity. Their test statistic is given by

$$
T V_{m n}=H V_{m n}
$$

where $H V_{m n}$ is Vasicek entropy estimator. For small values of the test statistic $T V_{m n}$, the uniformity hypothesis is rejected.

Alizadeh Noughabi (2017b) considered different entropy estimators and proposed some entropy based tests of uniformity. His tests are

$$
\begin{aligned}
T V E_{m n} & =H V E_{m n}, \\
T C_{m n} & =H C_{m n}, \\
T Y_{m n} & =H Y_{m n}, \\
T A_{m n} & =H A_{m n}, \\
T N_{m n} & =H N_{m n},
\end{aligned}
$$

where $H V E_{m n}, H C_{m n}, H Y_{m n}, H A_{m n}$, and $H N_{m n}$ are Van Es (1992), Correa (1995), Yousefzadeh and Argham (2008), Alizadeh Noughabi (2010), Alizadeh Noughabi and Alizadeh Noughabi (2008) estimators, respectively.

\subsection{Test for Other Distributions}

Testing procedures based on the sample entropy have been proposed for some other distributions as well. Jammalamadaka and Lund (2000) introduced a GOF test for von Mises distribution. For Inverse Gaussian distribution, Mudholkar and Tian (2002) proposed a test based on maximum entropy and then Alizadeh Noughabi and Vexler (2016) introduced tests for IG distribution based on the empirical likelihood ratio and minimum discrimination information (MDI) estimators. Recently, Alizadeh Noughabi and Jarrahiferiz (2018) introduced tests for the IG distribution based on correcting entropy estimators. Choi and Kim (2006), based on the maximum entropy of Laplace distribution, proposed a GOF test for this distribution. Then, Alizadeh Noughabi and Park (2016), based on correcting moments of entropy estimators, constructed tests for Laplace distribution and proposed some tests which were powerful. Perez-Rodriguez et al. (2009) proposed a test for Gumbel distribution based on the maximum entropy characterization. They used the moment and ML estimators for their test statistic. Baratpour and Khodadadi (2012), based the cumulative residual entropy, constructed a test for Rayleigh distribution. Finally, Mahdizadeh and Zamanzade (2017) proposed a GOF test for Cauchy distribution and obtained the power values of the proposed test and concluded that their test have a good power. 


\subsection{General Treatment of GOF Tests Based on Entropy}

Alizadeh Noughabi and Arghami (2013) investigated the general treatment of GOF tests based on entropy and proposed a general test statistic. Their test statistic can be obtained as follows. The KL discrimination information function is given by

$$
\begin{aligned}
D\left(f, f_{0} ; \theta\right) & =\int_{-\infty}^{\infty} f(x) \log \left(\frac{f(x)}{f_{0}(x ; \theta)}\right) d x \\
& =-H(f)-\int_{-\infty}^{\infty} f(x) \log \left\{f_{0}(x ; \theta)\right\} d x .
\end{aligned}
$$

Under the null hypothesis,

$$
\exists \theta \text { s.t. } f_{0}(x ; \theta)=f(x) \Leftrightarrow \exists \theta \text { s.t. } D\left(f, f_{0} ; \theta\right)=0 \Leftrightarrow \inf _{\theta \in \Theta} D\left(f, f_{0} ; \theta\right)=0 .
$$

Also under $H_{1}$,

$$
\forall \theta \quad f_{0}(x ; \theta) \neq f(x) \Leftrightarrow \forall \theta \quad D\left(f, f_{0} ; \theta\right)>0 \Leftrightarrow \inf _{\theta \in \Theta} D\left(f, f_{0} ; \theta\right)>0 .
$$

They used an estimate of $D_{\text {inf }}=\inf _{\theta \in \Theta} D\left(f, f_{0} ; \theta\right)$, denoted by $\hat{D}_{\text {inf }}$, where

$$
\hat{D}_{\text {inf }}=-H_{m n}-\sup _{\theta \in \Theta} \frac{1}{n} \sum_{i=1}^{n} \log \left(f_{0}\left(x_{i} ; \theta\right)\right) .
$$

Finally, their test statistic is stated as

$$
\begin{aligned}
\hat{D}_{\mathrm{inf}} & =-H V_{m n}-\sup _{\theta \in \Theta} \frac{1}{n} \sum_{i=1}^{n} \log \left(f_{0}\left(x_{i} ; \theta\right)\right) \\
& =-\frac{1}{n} \sum_{i=1}^{n} \log \left\{\frac{n}{2 m}\left(X_{(i+m: n)}-X_{(i-m: n)}\right)\right\}-\frac{1}{n} \sum_{i=1}^{n} \log \left(f_{0}\left(X_{i} ; \hat{\theta}\right)\right),
\end{aligned}
$$

where $\hat{\theta}$ is the maximum likelihood estimator of $\theta$. Then, they showed that the test based on $\hat{D}_{\text {inf }}$ is consistent and it is exact for location, scale and location-scale families.

Moreover, they showed that the minimum KL distance tests for the normality and exponentiality are equivalent to the entropy-based tests given by Vasicek (1976) and Ebrahimi et al. (1992), respectively. Generally, Alizadeh Noughabi and Arghami (2013) showed that all entropy-based tests introduced by the previous authors can be obtained by their general test.

Based on the above test statistic, Alizadeh Noughabi and Arghami (2013) constructed some GOF tests for Laplace, uniform, Weibull and beta distributions.

\subsection{Test for Symmetry Based on Entropy}

Let $E_{r_{1} \ldots r_{k}: n}$ represent the entropy of a set of order statistics $X_{\left(r_{1}: n\right)}, \ldots, X_{\left(r_{k}: n\right)}$. Then, we have

$$
E_{1: 1}=-\int_{-\infty}^{\infty} f(x) \log f(x) d x
$$


and

$$
E_{1: k}=-\log k-\int_{-\infty}^{\infty}\{\log f(x)+(k-1) \log (1-F(x))\} d F_{1: k}(x) .
$$

Park (1999) showed that

$$
\begin{aligned}
& E_{1: k}=1-\frac{1}{k}-\log k-\int_{-\infty}^{\infty} \log f(x) d F_{1: k}(x), \\
& E_{k: k}=1-\frac{1}{k}-\log k-\int_{-\infty}^{\infty} \log f(x) d F_{k: k}(x) .
\end{aligned}
$$

He also derived the sample estimates of $E_{1: k}$ and $E_{k: k}, k=1, \ldots, \infty$, by analogy to Vasicek (1976), as

$$
\begin{gathered}
\hat{E}_{1: k}=1-\frac{1}{k}-\log k+\frac{1}{n} \sum_{i=1}^{n} \log \left(\frac{n}{2 m}\left(X_{(i+m: n)}-X_{(i-m: n)}\right)\right) k\left(1-\frac{i}{n+1}\right)^{k-1}, \\
\hat{E}_{k: k}=1-\frac{1}{k}-\log k+\frac{1}{n} \sum_{i=1}^{n} \log \left(\frac{n}{2 m}\left(X_{(i+m: n)}-X_{(i-m: n)}\right)\right) k\left(\frac{i}{n+1}\right)^{k-1} .
\end{gathered}
$$

The sample estimates of $E_{1: k}$ and $E_{k: k}$ can be unified in the following general expression:

$$
\text { const. }+\frac{1}{n} \sum_{i=1}^{n} \log \left\{\frac{n}{2 m}\left(X_{(i+m: n)}-X_{(i-m: n)}\right)\right\} J\left(u_{i}\right),
$$

where $u_{i}=\frac{i}{n+1}, i=1, \ldots, n, J(u)$ is bounded and continuous and $J(u)=-J(1-u)$.

Park (1999) used the sample estimates of $E_{1: k}-E_{k: k}$ and introduced a characterization for symmetric.

Lemma 2.1. (Park, 1999) Let $D_{k}=E_{1: k}-E_{k: k}$, then $D_{k}=0$ for $k=1, \ldots, \infty$ if and only if $f$ is symmetric.

Park (1999) proved the above Lemma and used the estimate of $D_{k}$ to obtain a test statistic for testing normality against asymmetric alternatives. He considered the following general expression for estimating $D_{k}$ :

$$
D V(J, n, m)=\frac{1}{n} \sum_{i=1}^{n} \log \left(\frac{n}{2 m}\left(X_{(i+m: n)}-X_{(i-m: n)}\right)\right) J\left(u_{i}\right) .
$$

Habibi Rad and Arghami (2007) investigated the above test and evaluated its power properties. Abbasnejad and Mohammadi (2010), based on the above idea, constructed a symmetry test using Renyi information and then obtained power values of their test. Alizadeh Noughabi (2015) used the other entropy estimators and presented some nonparametric tests of symmetry based on the sample entropy of order statistics. His tests have this advantage that we do not need to estimate the center of the symmetry. Monte Carlo studies showed that the proposed tests were more powerful than previous ones against a variety of asymmetric alternative distributions. 


\subsection{Tests Based on Entropy for Ranked Set Samples (RSS)}

Ranked set sampling, originally developed by McIntyre (1952), combines simple random sampling (SRS) with the field investigator's professional knowledge and judgment to pick places to collect samples. Alternatively, field screening measurements can replace professional judgment when appropriate. The use of ranked set sampling increases the chance that the collected samples will yield representative measurements. This results in better estimates of the mean as well as improved performance of many statistical procedures such as testing for compliance with risk-based or backgroundbased (reference-based) standards.

Mahdizadeh and Arghami (2010) investigated the efficiency of ranked set sampling in entropy estimation and GOF testing for the inverse Gaussian law. Similarly, Mahdizadeh (2012) performed a similar study for the Laplace distribution. Al-Omari and Haq (2012) proposed GOF tests for the inverse Gaussian distribution based on new entropy estimation, using ranked set sampling and double ranked set sampling. Then, Mahdizadeh and Arghami (2013) improved the entropy based tests of uniformity using ranked set samples. Amini et al. (2016) and Zamanzade and Mahdizadeh (2017) proposed some entropy estimators based on ranked set sampling and compared them with other estimators based on SRS. Alizadeh Noughabi (2017a) proposed seven normality tests based on RSS and compared them with the similar tests based on SRS.

\subsection{Tests Based on Entropy for Independence}

A test of independence determines whether two factors are independent or not. This test determines if there is a relationship between two categorical variables in the population. It is called a test of independence because "no relationship" means "independent." If there is a relationship between the two variables in the population, then they are dependent.

Some authors including Sharifdoost et al. (2009), Fernandes and Neri (2010), Onken et al. (2012), and Lee and Kim (2017) proposed tests for independence based on entropy. They used the KL information and constructed a test statistic for independence.

\subsection{Test Based on Entropy for Censored Data (Type II, Progressive, Hybrid, etc.)}

The estimation of $H(f)$ based on censored samples has been done by Park (2005). If

$$
X_{(1: n)}, X_{(2: n)}, \ldots, X_{(r: n)}
$$

are the first $r$ order statistics of a random sample of size $n$, then entropy of these data is defined as

$$
H_{1, \ldots, r: n}=-\int_{-\infty}^{\infty} \ldots \int_{-\infty}^{x_{(2: n)}} f_{1, \ldots, r: n} \log f_{1, \ldots, r: n} d x_{(1: n)} \ldots d x_{(r: n)},
$$


where $f_{1, \ldots, r: n}$ is the joint probability density function of $X_{(1: n)}, \ldots, X_{(r: n)}$.

Park (2005) showed that

$$
H_{1, \ldots, r: n}=-(\log n+\ldots+\log (n-r+1))+n \bar{H}_{1, \ldots, r: n}
$$

where

$$
\bar{H}_{1, \ldots, r: n}=\frac{r}{n}-\int_{-\infty}^{\infty}\left(1-F_{r: n-1}\right) f(x) \log h(x) d x,
$$

and $h(x)$ is the hazard function. He further showed that

$$
\bar{H}_{1, \ldots, r: n}=-E\left(\int_{0}^{U_{r: n-1}} \log \left(\frac{d}{d p} F^{-1}(p)\right) d p\right)-E\left(\left(1-U_{r: n-1}\right) \log \left(1-U_{r: n-1}\right)\right),
$$

where $U_{r: n-1}$ is the $r$ th order statistic from a sample of size $n-1$, from the standard uniform distribution.

Based on the above expression, Park (2005) proposed an estimator of $\bar{H}_{1, \ldots, r: n}$ as

$$
H(n, m, r)=\frac{1}{n} \sum_{i=1}^{r} \log \left\{\frac{n}{2 m}\left(X_{(i+m: n)}-X_{(i-m: n)}\right)\right\}-\left(1-\frac{r}{n}\right) \log \left(1-\frac{r}{n}\right),
$$

where the window size $m$ is a positive integer less than or equal to $r / 2$, and $X_{(i: n)}=X_{(1: n)}$ if $i<1, X_{(i: n)}=X_{(r: n)}$ if $i>r$. Park (2005) then established the consistency of $H(n, m, r)$ for the population entropy.

Subsequently, some other authors also considered the problem of entropy estimation under different censored schemes. For example, Balakrishnan et al. (2007) estimated the joint entropy of a sample under progressive type-II censoring. Morrabi and Razmkhah (2009) obtained the entropy under hybrid censoring schemes, but did not consider the estimation of entropy.

Let $G(x ; \theta)$ be a parametric family of distributions with probability density function $g(x ; \theta)$. Then, the hypothesis of interest is

$$
H_{0}: f(x)=g(x ; \theta), \quad \text { for some } \theta \in \Omega,
$$

versus

$$
H_{1}: f(x) \neq g(x ; \theta), \quad \text { for any } \theta \in \Omega \text {. }
$$

To discriminate between the two hypotheses $H_{0}$ and $H_{1}$, the KL information can be used. If $f$ actually belongs to $G$, the minimal value of the KL information is zero and if $f$ does not belong to $G$, the minimal KL information is strictly positive. Therefore, a GOF test can be constructed which would reject $H_{0}$ for large value of $I_{1, \ldots, r: n}(f, g ; \theta)$, 
where

$$
\begin{aligned}
I_{1, \ldots, r: n}(f, g ; \theta) & =\int_{-\infty}^{\infty} f_{1, \ldots, r: n}(x) \log \frac{f_{1, \ldots, r: n}(x)}{g_{1, \ldots, r: n}(\theta)} d x \\
= & \int_{-\infty}^{\infty} f_{1, \ldots, r: n}(x) \log f_{1, \ldots, r: n}(x) d x-\int_{-\infty}^{\infty} f_{1, \ldots, r: n}(x) \log g_{1, \ldots, r: n}(x ; \theta) d x \\
& =-H_{1, \ldots, r: n}-\int_{-\infty}^{\infty} f_{1, \ldots, r: n}(x) \log g_{1, \ldots, r: n}(x ; \theta) d x,
\end{aligned}
$$

where $H_{1, \ldots, r: n}$ is the joint entropy of $X_{(1: n)}, \ldots, X_{(r: n)}$.

Park (2005) approximated $I_{1, \ldots, r: n}(f, g ; \theta)$ by

$$
I_{1, \ldots, r: n}(f, g ; \theta)=-n \bar{H}_{1, \ldots, r: n}-\sum_{i=1}^{r} \log g\left(x_{(i: n)} ; \theta\right)-(n-r) \log \left(1-G\left(x_{(r: n)} ; \theta\right)\right),
$$

and based on $I_{1, \ldots, r: n}(f, g ; \theta) / n$ proposed the following test statistic:

$$
T(n, m, r)=-H(n, m, r)-\frac{1}{n}\left(\sum_{i=1}^{r} \log g\left(x_{(i: n)} ; \hat{\theta}\right)+(n-r) \log \left(1-G\left(x_{(r: n)} ; \hat{\theta}\right)\right)\right),
$$

where $\hat{\theta}$ is an estimate of $\theta$ and $H(n, m, r)$ is Park's estimate of $\bar{H}_{1, \ldots, r: n}$.

Alizadeh Noughabi and Balakrishnan (2015) proposed to estimate $I_{1, \ldots, r: n}(f, g ; \theta)$ by

$$
T A(n, m, r)=-H(n, m, r)-H A(n, m, r),
$$

where $H(n, m, r)$ is Park's estimate of $\bar{H}_{1, \ldots, r: n}$ and $H A(n, m, r)$ is the semi-parametric estimate of second part of KL information, given by

$$
H A(n, m, r)=\sum_{i=1}^{r} \log \left\{\frac{G\left(X_{(i+m: n)} ; \hat{\theta}\right)-G\left(X_{(i-m: n)} ; \hat{\theta}\right)}{X_{(i+m: n)}-X_{(i-m: n)}}\right\}+(n-r) \log \left(1-G\left(x_{(r: n)} ; \hat{\theta}\right)\right) .
$$

Consequently, the proposed test statistic based on $I_{1, \ldots, r: n}(f, g ; \theta) / n$ is

$$
\begin{aligned}
T A(n, m, r) & =\frac{1}{n} \sum_{i=1}^{r} \log \left\{\frac{2 m / n}{X_{(i+m: n)}-X_{(i-m: n)}}\right\}+\left(1-\frac{r}{n}\right) \log \left(1-\frac{r}{n}\right) \\
& -\frac{1}{n} \sum_{i=1}^{r} \log \left\{\frac{G\left(X_{(i+m: n)} ; \hat{\theta}\right)-G\left(X_{(i-m: n)} ; \hat{\theta}\right)}{X_{(i+m: n)}-X_{(i-m: n)}}\right\}-\left(\frac{n-r}{n}\right) \log \left(1-G\left(x_{(r: n)} ; \theta\right)\right) \\
= & -\frac{1}{n} \sum_{i=1}^{r} \log \left\{\frac{n}{2 m}\left(G\left(X_{(i+m: n)} ; \hat{\theta}\right)-G\left(X_{(i-m: n)} ; \hat{\theta}\right)\right)\right\}+\left(1-\frac{r}{n}\right) \log \left(\frac{1-r / n}{\left.1-G\left(x_{(r: n)}\right) \hat{\theta}\right)}\right),
\end{aligned}
$$

where $G$ denotes the distribution function of $g$ under the null hypothesis, $m$ is a positive integer such that $m$ less than or equal to $\frac{r}{2}$, and $X_{(1: n)}, \ldots, X_{(r: n)}$ are the order statistics. Also, $\hat{\theta}$ can be any equivariant estimate of $\theta$.

Other authors such as Lim and Park (2007), Balakrishnan et al. (2007), Yousefzadeh and Arghami (2008), Habibi Rad et al. (2011), Alizadeh Noughabi (2017c), Alizadeh Noughabi (2019), and Alizadeh Noughabi and Chahkandi (2018) introduced some tests under different censoring schemes based on entropy or censored KL Information. 


\subsection{Tests Based on Entropy for the Residual Life}

In survival or reliability studies, the mean residual life or life expectancy is an important characteristic of the model. If $X$ is a nonnegative random variable representing the life of a component having distribution function $\mathrm{F}$, the mean residual life function or life expectancy function at age $x$ is defined by

$$
\varepsilon(x)=E(X-x \mid X>x) .
$$

Ebrahimi (1998) considered the problem of testing GOF for exponentiality of the remaining life of a system at a certain age. He developed a test statistic for the above problem based on the dynamic version of KL information. Also, Ebrahimi (2001) proposed a test statistic for uniformity of the remaining life of a system at a certain age based on the dynamic version of KL information. Recently, Chahkandi and Alizadeh Noughabi (2016) proposed a new test for exponentiality of the residual life of a system at a certain age. They investigated the properties of the test statistic and then compared the power of the new test with Ebrahimi's test.

\subsection{Test Based on Entropy for Fuzzy Data}

The theory of fuzzy sets and systems has been widely employed in many pattern analysis applications. It is a well known tool for formulation and analysis of imprecise and subjective concepts. The common tests of fit are based on precise data. But, in practical problems, different kinds of uncertainty exist, especially in problems with imprecise data. In other words, the data sometimes cannot be recorded precisely. For example, because of the fluctuation of a river, the water level cannot be measured in an exact way. Then, we say that the water level is around 30 meters, for example. The phrase 'around 30 meters' can be considered as a fuzzy number. Therefore, the fuzzy sets theory is an appropriate tool for analyzing the imprecise data.

Given a fuzzy random sample $\left(\tilde{X}_{1}, \tilde{X}_{2}, \ldots, \tilde{X}_{n}\right)$ from a continuous probability distribution $F$ with a density $f(x)$, the hypothesis of interest is

$$
\begin{aligned}
& H_{0}:\left(\tilde{X}_{1}, \tilde{X}_{2}, \ldots, \tilde{X}_{n}\right) \text { is a sample from normal distribution, } \\
& \text { versus } \\
& H_{1}:\left(\tilde{X}_{1}, \tilde{X}_{2}, \ldots, \tilde{X}_{n}\right) \text { is not a sample from a normal population, }
\end{aligned}
$$

where $\mu$ and $\sigma$ are unspecified.

According to Alizadeh Noughabi and Akbari (2016) the fuzzy random variable $\tilde{X}$ is fuzzy normal if and only if its $\alpha$-pessimistic is normal for all values of $\alpha \in[0,1]$. Based on this definition Alizadeh Noughabi and Akbari (2016) proposed the following test statistics.

1- The Kolmogorov-Smirnov type test statistic :

$$
K S=\int_{0}^{1} \sup _{\tilde{y}_{\alpha i}}\left|F_{n}\left(\tilde{y}_{\alpha i}\right)-Z_{\alpha i}\right| d \alpha,
$$


which is approximated by

$$
K S=\frac{1}{100} \sum_{\alpha=.01}^{1} \max \left\{\max _{1 \leq i \leq n}\left\{\frac{i}{n}-Z_{\alpha i}\right\}, \max _{1 \leq i \leq n}\left\{Z_{\alpha i}-\frac{i-1}{n}\right\}\right\} .
$$

2- The Cramer-von Mises type test statistic :

$$
C M=\int_{0}^{1} \int_{-\infty}^{\infty}\left(F_{n}\left(\tilde{y}_{\alpha i}\right)-\Phi\left(\tilde{y}_{\alpha i}\right)\right)^{2} d \Phi(\tilde{y}) d \alpha
$$

which we approximate it by

$$
C M=\frac{1}{100} \sum_{\alpha=.01}^{1}\left(\frac{1}{12 n}+\sum_{i=1}^{n}\left(Z_{\alpha i}-\frac{2 i-1}{2 n}\right)^{2}\right)
$$

3- The Kuiper type test statistic :

$$
V=\frac{1}{100} \sum_{\alpha=.01}^{1}\left\{\max _{1 \leq i \leq n}\left\{\frac{i}{n}-Z_{\alpha i}\right\}+\max _{1 \leq i \leq n}\left\{Z_{\alpha i}-\frac{i-1}{n}\right\}\right\}
$$

4- The Anderson-Darling type test statistic :

$$
A D=\frac{1}{100} \sum_{\alpha=.01}^{1}\left(-n-\frac{\sum_{i=1}^{n}(2 i-1)\left\{\ln \left(Z_{\alpha i}\right)+\ln \left(1-Z_{\alpha(n-i+1)}\right)\right\}}{n}\right)
$$

5- The Shapiro-Wilk type test statistic :

$$
W=\frac{1}{100} \sum_{\alpha=.01}^{1} \frac{\left(\sum_{i=1}^{[n / 2]} a_{(n-i+1)}\left(\tilde{y}_{\alpha(n-i+1)}-\tilde{y}_{\alpha i}\right)\right)^{2}}{\sum_{i=1}^{n}\left(\tilde{y}_{\alpha i}-\bar{y}_{\alpha}\right)^{2}} .
$$

Through a Monte Carlo simulation, they computed the power values of the tests under 20 alternatives. Similarly, Zendehdel et al. (2018) introduced some exponentiality tests for fuzzy data. 


\subsection{Tests Based on Cumulative Residual Entropy (CRE)}

Rao et al. (2004) proposed the cumulative residual entropy (CRE) of a nonnegative random variable, which covers some problems of Shannon entropy, as

$$
C R E(F)=-\int_{0}^{\infty} \bar{F}(x) \log \bar{F}(x) d x,
$$

where $\bar{F}(x)=1-F(x)$ is the survival function.

Drissi et al. (2008) extended the definition of CRE to the whole real line. CRE has its merit that it is well defined on the empirical distribution function. Hence, its nonparametric estimate can be instantly obtained as

$$
\operatorname{CRE}\left(F_{n}\right)=-\int_{0}^{\infty} \bar{F}_{n}(x) \log \bar{F}_{n}(x) d x,
$$

where $F_{n}(x)$ is the empirical distribution function.

The cumulative residual KL information (Baratpour and Habibi Rad, 2012) has been defined in relation with CRE as

$$
C R K L(F: G)=\int_{0}^{\infty} \bar{F}(x) \log \frac{\bar{F}(x)}{\bar{G}(x)} d x-(E(X)-E(Y)) .
$$

The functions $\bar{F}(x)$ and $\bar{G}(x)$ are the survival functions of nonnegative continuous random variables $X$ and $Y$, respectively.

CRKL has the nonnegativity and characterization properties as a distance function between two distributions, but it is not symmetric. CRKL is also well defined on the empirical distribution function, and its estimate based on the empirical distribution function has been studied as a GOF test for exponentiality by Baratpour and Habibi $\operatorname{Rad}$ (2012). Because CRKL is defined on the nonnegative random variables, so that $\int_{0}^{\infty} \bar{F}(x) d x$ can be defined as $E(X)$, its application has been limited to the semi-infinite intervals.

Park et al. (2018) extend the application of CRKL to the whole real line as

$$
C R K L(F: G)=\int_{-\infty}^{\infty} \bar{F}(x) \log \frac{\bar{F}(x)}{\bar{G}(x)} d x-\int_{-\infty}^{\infty}(\bar{F}(x)-\bar{G}(x)) d x .
$$

They also considered the extension of the cumulative KL information (Park et al. (2012)) as

$$
C K L(F: G)=\int_{-\infty}^{\infty} F(x) \log \frac{F(x)}{G(x)} d x-\int_{-\infty}^{\infty}(F(x)-G(x)) d x .
$$

CKL gives more weights on later difference while CRKL gives more weights on earlier difference. In this sense, it is better to consider CRKL in measuring the distance 
between lifetime distributions because CRKL is more sensitive to earlier difference. If we consider distribution functions supported on the whole real line, it may be proper to consider a general cumulative KL information (GCKL) defined as

$$
\operatorname{GCKL}_{\alpha}(F: G)=\alpha \operatorname{CKL}(F: G)+(1-\alpha) \operatorname{CRKL}(F: G), 0 \leq \alpha \leq 1 .
$$

The choice of $\alpha$ depends on whether to put more weight on earlier difference or later difference.

Park et al. (2018) considered a normal distribution as an example of distribution functions supported on the whole real line, and proposed GOF test statistics based on $C R K L, C K L$ and $G C K L_{\alpha}$, respectively. Then, They compared their performances with some competing test statistics based on the empirical distribution function, including the Kolmogorov-Smirnov, Cramer von Mises, and Anderson Darling tests.

Some other authors used the CRE and constructed tests for exponential and normal distributions. See for example, Baratpour and Habibi Rad (2012, 2016), Park et al. (2012), Park and Lim (2015), Park and Pakyari (2015), and Zardasht et al. (2015).

\subsection{Tests Based on Other Divergences}

Shannon entropy and KL divergence are perhaps the two most fundamental quantities in information theory and its applications. Because of their success, there have been many attempts to generalize these concepts, and in the literature one will find numerous entropy and divergence measures. Most of these quantities have never found any applications, and almost none of them have found an interpretation in terms of coding. The most important exceptions are the Renyi entropy and Renyi divergence. Renyi divergence is related to Renyi entropy much like KL divergence is related to Shannon's entropy, and comes up in many settings. It was introduced by Renyi as a measure of information that satisfies almost the same axioms as KL divergence, and depends on a parameter that is called its order. In particular, the Renyi divergence of order 1 equals the KL divergence.

The following authors considered other entropies (for example, Renyi, Verma) or Phi-Divergence and constructed tests for statistical distributions, Pasha et al. (2008), Abbasnejad (2011), Lequesne (2015), Abbasnejad (2016), Alizadeh Noughabi and Balakrishnan (2016). These authors compared their tests with some other tests, such as entropy-based tests, and concluded that for some alternatives their tests have a higher power than their competitors.

\section{Simulation Study}

In order to perform a simulation study in this context, since the distribution of the test statistics under the null hypothesis are complicated for analytical evaluation, the critical values of the test statistics are computed using Monte Carlo simulation. We use the following steps to determine the critical values of the test statistics. 
1. Generate a sample $X_{1}, \ldots, X_{n}$ of size $\mathrm{n}$ from the null distribution;

2. Calculate the test statistics based on the sample $X_{1}, \ldots, X_{n}$;

3. Repeat Steps 1-2 a large number of times and then determine the quantile of the statistics.

The obtained critical values of the test statistics can be found in the corresponding articles. Through Monte Carlo simulation, the power values of the tests can be computed. Since the tests based on the empirical distribution function are commonly used in practice, the authors compared the power values of the entropy-based tests with the power values of the EDF-based tests. For more details about these tests, see D'Agostino and Stephens (1986).

In power comparisons of the tests, authors consider various alternatives. For example, in exponentiality tests, the considered alternatives include densities with decreasing failure rates (DFR), increasing failure rates (IFR), as well as models with unimodal failure rate (UFR) and bathtub failure rate (BFR) functions.

Under the considered alternatives, the power values of the tests can be computed by means of Monte Carlo simulations. Under each alternative, several samples of size $n$ are generated and the test statistics are calculated. Then power values of the corresponding test are computed by the frequency of the event "the statistic is in the critical region". To save space, the power values of the tests are not reported and the results of a simulation study can be observed in the following papers.

The normality tests are compared by Esteban et al. (2001), Senoglu and Surucu (2004), Alizadeh Noughabi (2010), Alizadeh Noughabi and Arghami (2011c, 2011f, 2012, 2013) and Zamanzade and Arghami (2011, 2012). The GOF tests for exponentiality are compared by Alizadeh Noughabi and Arghami (2011a, b, d, e), and Zamanzade and Arghami (2011). The uniformity tests are compared by Alizadeh Noughabi (2017a).

In all of the above papers, it is shown that none of the tests is optimum for all alternatives but the entropy-based tests generally have a better performance than the competitors and therefore these tests can be confidently used in practice.

\section{Conclusions}

In this paper, we collected the developments on GOF based on entropy during the last 50 years, from the very first origins until the most recent advances for different data and models. We explained different entropy-based GOF tests constructed by authors from the beginning to now. All works proposed by authors in this subject have been expressed.

The properties of the tests are stated and then the critical values are discussed. Finally, method of computing the power values of the considered tests against different alternatives was presented. Based on simulation study performed by some authors, 
we found that none of the tests is optimum for all alternatives. However, we observed that the entropy-based tests have a higher power than competitors against some alternatives and are superior to the other tests. For example, the entropy-based tests for the exponential distribution have high power against IFR (increasing failure rate) alternatives. Therefore, the choice of a specific test depends on the alternative distribution.

In general, based on the simulation study performed by authors, we can say that the power values of entropy-based tests are reasonable and acceptable and therefore these tests can be confidently used in practice.

\section{References}

Abbasnejad, M. (2016), Modified estimators of Renyi entropy with application in testing exponentiality based on transformed data. ISTATISTIK: Journal of the Turkish Statistical Association, 9, 42-55.

Abbasnejad, M., and Mohammadi, D. (2010), A Test for symmetric distributions based on Renyi entropy. Journal of Statistical Science, 4, 21-33.

Alizadeh Noughabi, H. (2019), A new estimator of Kullback-Leibler information and its application in goodness of fit tests, Journal of Statistical Computation and Simulation, 89, 1914-1934.

Alizadeh Noughabi, H. (2017a), Efficiency of ranked set sampling in tests for normality. Journal of Statistical Computation and Simulation 87, 956-965.

Alizadeh Noughabi, H. (2017b), Entropy based tests of uniformity: A Monte Carlo power comparison. Communication in Statistics: Simulation and Computation, 46, 12661279 .

Alizadeh Noughabi, H. (2017c), Testing exponentiality based on Kullback-Leibler information for progressively Type II censored data. Communications in StatisticsSimulation and Computation, 46, 7624-7638.

Alizadeh Noughabi, H. (2015), Tests of symmetry based on the sample entropy of order statistics and power comparison. Sankhya B: The Indian Journal of Statistics, 77, 240-255.

Alizadeh Noughabi, H. (2015a), Entropy estimation using numerical methods. Annals of Data Science, 2, 231-241.

Alizadeh Noughabi, H. (2015b), On the estimation of Shannon entropy. Journal of Statistical Research of Iran (JSRI), 12, 57-70.

Alizadeh Noughabi, H. (2010), A new estimator of entropy and its application in testing normality. Journal of Statistical Computation and Simulation, 80, 1151-1162. 
Alizadeh Noughabi, H., and Akbari, M. GH. (2016), Testing normality based on fuzzy data. International Journal of Intelligent Technologies and Applied Statistics, 9, 37-52.

Alizadeh Noughabi, H., and Alizadeh Noughabi, R. (2008), Power comparisons of goodness of fit tests based on entropy with other methods. Journal of Statistical Science, 2, 97-113.

Alizadeh Noughabi, H., and Arghami, N. R. (2013), A Goodness-of-fit tests based on correcting moments of entropy estimators. Communications in Statistics-Simulation and Computation, 42(3), 499-513.

Alizadeh Noughabi, H., and Arghami, N. R. (2012), General treatment of goodness-offit tests based on Kullback-Leibler information. Journal of Statistical Computation and Simulation, 83, 1556-1569.

Alizadeh Noughabi, H., and Arghami, N. R. (2011a), Goodness of fit tests based on correcting moments of entropy estimators. Communication in Statistics: Simulation and Computation, 42, 499-513.

Alizadeh Noughabi, H., and Arghami, N. R. (2011b), Monte Carlo comparison of five exponentiality tests using different entropy estimates. Journal of Statistical Computation and Simulation, 81, 1579-1592.

Alizadeh Noughabi, H., and Arghami, N. R. (2011c), Monte Carlo comparison of seven normality tests. Journal of Statistical Computation and Simulation, 81, 965-972.

Alizadeh Noughabi, H., and Arghami, N. R. (2011d), Testing exponentiality using transformed data. Journal of Statistical Computation and Simulation,81, 511-516.

Alizadeh Noughabi, H., and Arghami, N. R. (2011e), Testing exponentiality based on characterizations of the exponential distribution. Journal of Statistical Computation and Simulation, 81, 1641-1651.

Alizadeh Noughabi, H., and Arghami, N. R. (2011f), Testing normality using transformed data. Communication in Statistics: Theory and Methods, 42, 3065-3075.

Alizadeh Noughabi, H., and Arghami, N. R. (2010), A new estimator of entropy. Journal of the Iranian Statistical Society (JIRSS), 9, 53-64.

Alizadeh Noughabi, H., and Balakrishnan, N. (2016), Tests of goodness of fit based on Phi divergence. Journal of Applied Statistics, 43, 412-429.

Alizadeh Noughabi, H., and Balakrishnan, N. (2015), Goodness of fit using a new estimate of Kullback-Leibler information based on type II censored data. IEEE Transactions on Reliability, 64, 627-635.

Alizadeh Noughabi, H. and Chahkandi, M. (2018), Testing the validity of the exponential model for hybrid Type-I censored data. Communications in StatisticsTheory and Methods, 47, 5770-5778. 
Alizadeh Noughabi, H. and Jarrahiferiz, J. (2018), Moments of nonparametric probability density functions of entropy estimators applied to testing the inverse Gaussian distribution. Journal of Statistical Computation and Simulation, 88, 3217-3229.

Alizadeh Noughabi, H., and Park, S. (2016), Tests of fit for the Laplace distribution based on correcting moments of entropy estimators. Journal of Statistical Computation and Simulation, 86, 2165-2181.

Alizadeh Noughabi, H., and Vexler, A. (2016), An efficient correction to the densitybased empirical likelihood ratio goodness of fit test for the Inverse Gaussian distribution. Journal of Applied Statistics, 43, 2988-3003.

Al-Omari, A. I., and Haq, A. (2012), Goodness-of-fit testing for the inverse Gaussian distribution based on new entropy estimation using ranked set sampling and double ranked set sampling. Environmental Systems Research, 1, 1-8.

Amini, M., Mehdizadeh, M. and Arghami, N. R. (2016), Improved estimator of the entropy and goodness of fit tests in ranked set sampling. arXiv:1106.1733v1 [stat.CO]

Anderson, T. W., and Darling, D. A. (1954), A test of goodness of fit. Journal of American Statistical Association, 49, 765-769.

Balakrishnan, N., Habibi Rad, A. and Arghami, N. R. (2007), Testing exponentiality based on Kullback-Leibler information with progressively type-II censored data. IEEE Transactions on Reliability, 56, 301-307.

Baratpour S., and Khodadadi F. (2012), A cumulative residual entropy characterization of the Rayleigh distribution and related goodness-of-fit test. Journal of Statistical Research of Iran, 9, 115-131.

Baratpour, S., and Habibi Rad, A. (2012), Testing goodness-of-fit for exponential distribution based on cumulative residual entropy. Communications in StatisticsTheory and Methods, 41, 1387-1396.

Baratpour, S., and Habibi Rad, A. (2016), Exponentiality test based on the progressive type II censoring via cumulative entropy. Communications in Statistics-Theory and Methods, 45, 2625-2637.

Beirlant, J., Dudewica, E. J., Gyorfi, L., and Van Der Meulin, E. C. (1997), Nonparametric entropy: an overview. International Journal of Mathematics and Mathematical Sciences, 6, 17-39.

Bonett, D. G., and Seier, E. (2002), A test of normality with high uniform power. Computational Statistics and Data Analysis, 40, 435-445.

Bowman, A. W. (1992), Density based tests for goodness of fit. Journal of Statistical Computation and Simulation, 40, 1-13. 
Chahkandi, M., and Alizadeh Noughabi, H. (2016), Testing exponentiality of the residual life, based on dynamic cumulative residual entropy. Statistics and Probability Letters, 117, 1-11.

Chen, L., and Shapiro, S. S. (1995), An alternative test for normality based on normalized spacings. Journal of Statistical Computation and Simulation, 53, 269-287.

Choi, B. (2008), Improvement of goodness-of-fit test for normal distribution based on entropy and power comparison. Journal of Statistical Computation and Simulation, 78, 781-788.

Choi, B., and Kim, K. (2006), Testing goodness-of-fit for Laplace distribution based on maximum entropy. Statistics, 40, 517-531.

Choi, B., Kim, K., and Song, S. H. (2004), Goodness-of-fit test for exponentiality based on Kullback-Leibler information. Communication in Statistics: Simulation and Computation, 33, 525-536.

Correa, J. C. (1995), A new estimator of entropy. Communications in Statistics-Theory and Methods, 24, 2439-2449.

D'Agostino R. B., and Pearson E. S. (1973), Tests for departure from normality: Empirical results for the distributions of b2 and $\sqrt{ } \mathrm{b} 1$. Biometrika, 60, 613-622.

D'Agostino, R. B., and Stephens, M. A. (1986), Goodness-of-Fit Techniques, New York: Marcel Dekker.

Dobrushin, R. L., (1958), Simplified method of experimental estimate of entropy of stationary sequence. Theory Probability and its Applications, 3, 462-464.

Doornik, J. A., and Hansen, H. (1994), An omnibus test or univariate and multivariate normality, Working Paper, Nuffield College, Oxford.

Dudewicz, E. J., and van der Meulen, E. C. (1987), Empirical entropy, a new approach to non-parametric entropy estimation, In: Puir, M. L., Vilaplana, J. P. and Wrtz, W. (Eds.), New Perspectives in Theoretical and Applied Statistics. Wiley, New York, 202-207.

Dudewicz, E. J., and van der Meulen, E. C. (1981), Entropy-Based Tests of Uniformity. Journal of the American Statistical Association, 76, 967-974.

Drissi, N., Chonavel, T., and Boucher, J. M. (2008), Generalized cumulative residual entropy for distributions with unrestricted supports, Research Letters in Signal Processing, Article ID 790607, 5 pages, 2008. doi:10.1155/2008/790607.

Ebrahimi, N. (2001), Testing for Uniformity of the Residual Life Time Based on Dynamic Kullback-Leibler Information. Annals of the Institute of Statistical Mathematics, 53, 325-337. 
Ebrahimi, N. (1998), Testing exponentiality of the residual life, based on dynamic Kullback-Leibler information. IEEE Transactions on Reliability, 47, 197-201.

Ebrahimi, N., Pflughoeft, K., and Soofi, E. S. (1994), Two measures of sample entropy. Statistics and Probability Letters, 20, 225-234.

Ebrahimi, N., Soofi., E. S., Habibullah, M. (1992), Testing exponentiality based on Kullback-Leibler information. Journal of the Royal Statistical Society B, 54, 739-748.

Epps, T. W., and Pulley, L. B. (1983), A test for normality based on the empirical characteristic function. Biometrika, 70, 723-726.

Esteban, M. D., Castellanos, M. E., Morales, D., and Vajda, I. (2001), Monte Carlo comparison of four normality tests using different entropy estimates. Communications in Statistics-Simulation and computation, 30, 761-285.

Fernandes, M., and Neri, B. (2010), Nonparametric entropy-based tests of independence between stochastic processes. Econometric Reviews, 29, 276-306.

Filliben, J. J. (1975), The probability plot correlation coefficient test for normality. Technometrics, 17, 111-117.

Gel, Y. R., and Gastwirth, J. L. (2008), A robust modification of the Jarque-Bera test of normality. Economics Letters, 99, 30-32.

Gokhale, D. V., (1983), On entropy-based goodness-of-fit tests. Computational Statistics and Data Analysis, 1, 157-165.

Goria, M. N., Leonenko N. N., Mergel V. V., and Novi Inverardi, P. L. (2005), A new class of random vector entropy estimators and its applications in testing statistical hypotheses. Journal of Nonparametric Statistics, 17, 277-297.

Greenwood, C., and Nikulin, M. S. (1996), A Guide to Chi-Squared Testing, New York: Wiley, ISBN 0-471-55779-X.

Gurevich, G., and Vexler, A. (2011), A Two-sample empirical likelihood ratio test based on samples entropy. Statistics and Computing, 21, 657-670.

Gurevich, G., and Davidson, A. (2008), Standardized forms of Kulback-Leibler information based statistics for normality and exponentiality. Computer Modelling and New Technologies, 12, 14-25.

Habibi Rad, A., and Arghami, N. R. (2007), Test for symmetry of distribution based on the entropy. Journal of Statistical Science, 1, 109-120.

Habibi Rad, A., Yousefzadeh, F. and Balakrishnan, N. (2011), Goodness-of-fit test based on Kullback-Leibler information for progressively type-II censored data. IEEE Transactions on Reliability, 60, 570-579. 
Hall, P. and Morton, S.C., (1993), On the estimation of entropy. Annals Institute of Statistical and Mathematics, 45, 69-88.

Jammalamadaka, S. R., and Lund, U. J. (2000), An entropy-based test for goodness of fit of the von Mises distribution (with U. Lund). Journal of Statistical Computation and Simulation, 67, 319-332.

Jarque, C., and Bera, A. (1980), Efficient tests for normality, homoscedasticity and serial independence of regression residuals. Economics Letters, 6, 255-259.

Joe, H., (1989), Estimation of entropy and other functionals of a multivariate density. Annals Institute of Statistical and Mathematics, 41, 683-697.

Kolmogorov, A. N. (1933), Sulla Determinazione Empirica di une legge di Distribuzione. Giornale dell'Intituto Italiano degli Attuari, 4, 83-91.

Kuiper, N. H. (1960), Tests concerning random points on a circle. Proceedings of the Koninklijke Nederlandse Akademie van Wetenschappen, Series A, 63, 38-47.

Lee, S. (2013), A maximum entropy type test of fit: Composite hypothesis case. Computational Statistics and Data Analysis, 57, 59-67.

Lee, S., and Kim, M. (2017), On entropy test for conditionally heteroscedastic locationscale time series models. Entropy, 19, 388-398.

Lequesne, J. (2015), A goodness-of-fit test of student distributions based on Rényi entropy. AIP Conference Proceedings, 1641, 487-494.

Lilliefors, H. (1967), On the Kolmogorov-Smirnov test for normality with mean and variance unknown. Journal of the American Statistical Association, 62, 399-402.

Lim, J., and Park, S. (2007), Censored Kullback-Leibler information and goodness-of-fit test with type II censored data. Journal of Applies Statistics, 34, 1051-1064.

Mahdizadeh M. (2012), On the use of ranked set samples in entropy based test of fit for the Laplace distribution. Revista Colombiana de Estadística, 35, 443-455.

Mahdizadeh, M., and Zamanzade, E. (2017), New goodness of fit tests for the Cauchy distribution. Journal of Applied Statistics, 44, 1106-1121.

Mahdizadeh, M., and Arghami, N. R. (2010), Efficiency of ranked set sampling in entropy estimation and goodness-of-fit testing for the inverse Gaussian law. Journal of Statistical Computation and Simulation, 80, 761-774.

Mahdizadeh, M., and Arghami, N. R. (2013), Improved entropy based test of uniformity using ranked set samples, Statistics and Operations Research Transactions. SORT, 37, 3-18.

McIntyre, G. A. (1952), A method for unbiased selective sampling using ranked sets, Australian Journal of Agricultural Research. 3,385-390. 
Mises, R. von (1931), Wahrscheinlichkeitsrechnung und ihre Anwendung in der Statistik und theoretischen Physik. Leipzig and Vienna: Deuticke.

Mudholkar, G. S., and Tian, L. (2002), An entropy characterization of the inverse Gaussian distribution and related goodness-of-fit test. Journal of Statistical Planning and Inference, 102, 211-221.

Onken, A., Dragoi, V., and Obermayer, K. (2012), A maximum entropy test for evaluating higher-order correlations in spike counts. PLOS Computational Biology, $8,1-11$.

Park, S., Alizadeh Noughabi, H., and Kim, I. (2018), General cumulative KullbackLeibler information. Communications in Statistics-Theory and Methods, 47, 1551-1560.

Park, S., and Pakyari, R. (2015), Cumulative residual Kullback-Leibler information with the progressively type-II censored data. Statistics and Probability Letters, 106, 287-294.

Park, S. (2005), Testing exponentiality based on the Kullback-Leibler information. IEEE Transactions on Reliability, 54, 22-26.

Park, S. (1999), A goodness-of-fit test for normality based on the sample entropy of order statistics. Statistics and Probability Letters, 44, 359-363.

Park, S., and Park, D. (2003), Correcting moments for goodness of fit tests based on two entropy estimates. Journal of Statistical Computation and Simulation, 73, 685-694.

Park, S., and Lim, J., (2015), On censored cumulative residual Kullback-Leibler Information and goodness-of-fit test with Type-II censored data. Statistical Papers, $56,247-256$.

Park, S., Rao, M., and Shin, D. (2012), On cumulative residual Kullback-Leibler information. Statistics and Probability Letters, 82, 2025-2032.

Pasha, E., Kokabi, M., and Mohtashami, G. R. (2008), Goodness-of-fit tests based on divergence measures. Journal of Applied Mathematics and Informatics, 26, 177-189.

Perez-Rodriguez, P., Vaquera-Huerta, H., and Villasenor-Alva, J. (2009), A Goodnessof-Fit Test for the Gumbel Distribution Based on Kullback-Leibler Information. Communications in Statistics: Theory and Methods, 38,842-855.

Rahman M. M., and Govindarajulu Z. (1997), A modification of the test of Shapiro and Wilk for normality. Journal of Applied Statistics, 24, 219-235.

Rao, M., Chen, Y., Vemuri, B. C., Wang, F. (2004), Cumulative residual entropy: a new measure of information. IEEE Transactions on Information Theory, 50, 1220-1228.

Senoglu, B., and Surucu, B. (2004), Goodness of fit tests based on Kullback-Leibler information. IEEE Transactions on Reliability, 53,22-26. 
Shannon, C. E. (1948), A mathematical theory of communications. Bell System Technical Journal, 27, 379-423; 623-656.

Shannon, C. E. (1949), The mathematical theory of communications. Urbana: University of Illinois Press.

Shapiro, S. S., and Francia, R. S. (1972), An approximate analysis of variance test for normality. Journal of the American Statistical Association, 67, 215-216.

Shapiro, S. S., and Wilk, M. B. (1965), An analysis of variance test for normality (complete samples). Biometrika, 52, 591-611.

Sharifdoost, M., Nematollahi, N., and Pasha, E. (2009), Goodness-of-Fit test and test of independence by entropy. Journal of Mathematical Extension, 3, 43-59.

Song, K. S., (2000), Limit theorems for nonparametric sample entropy estimators. Statistics and Probability Letters, 49, 9-18.

Van Es, B. (1992), Estimating functionals related to a density by a class of statistic based on spacings. Scandinavian Journal of Statistics, 19, 61-72.

Vasicek, O. (1976), A test for normality based on sample entropy. Journal of the Royal Statistical Society B, 38, 54-59.

Vatutin, V. A. and Michailov, V. G. (1995), Statistical estimation of entropy of discrete random variables with large numbers of results. Russian Mathematical Surveys, 50, 963-976.

Vexler, A., and Gurevich, G. (2010), Empirical likelihood ratios applied to goodnessof-fit tests based on sample entropy. Computational Statistics and Data Analysis, 54, 531-545.

Vexler, A., Tsai, W. M., and Hutson, A. D. (2014), A simple density-based empirical likelihood ratio test for Independence. The American Statistician, 48, 158-169.

Watson, G. S. (1961), Goodness of fit tests on a circle. Biometrika, 48, 109-114.

Wieczorkowski, R., and Grzegorzewsky, P. (1999), Entropy estimators improvements and comparisons. Communications in Statistics-Simulation, 28, 541-567.

Yousefzadeh, F., and Arghami, N. R. (2008), Testing Exponentiality Based on Type II Censored Data and a New cdf Estimator. Communications in Statistics-Simulation and Computation, 37, 1479-1499.

Zamanzade, E., and Arghami, N. R. (2009), Normality and exponentiality tests based on new entropy estimators. Journal of Statistical Science, 2, 179-200.

Zamanzade, E., and Arghami, N. R. (2011), Goodness of fit test based on correcting moments of modified entropy estimator. Journal of Statistical Computation and Simulation, 81, 2077-2093. 
Zamanzade, E., and Arghami, N. R. (2012), Testing normality based on new entropy estimators. Journal of Statistical Computation and Simulation, 82, 1701-1713.

Zamanzade, E., and Mahdizadeh M. (2017), Entropy estimation from ranked set samples with application to test of fit. Revista Colombiana de Estadística, 40, 223-241.

Zardasht V., Parsi S., and Mousazadeh, M. (2015), On empirical cumulative residual entropy and a goodness-of-fit test for exponentiality. Statistical Papers, 56, 677-688.

Zendehdel, J., Rezaei, M., Akbari, M. GH., Zarei, R., and Alizadeh Noughabi, H. (2018), Testing exponentiality for imprecise data and its application. Soft Computing, 22, 3301-3312.

Zhang, J. (2002), Powerful goodness-of-fit tests based on the likelihood ratio, Journal of Royal Statistical Society, Series B, 64, 281-294. 